\title{
Stone-Wales defects in silicene: Formation, stability, and reactivity of defect sites
}

\author{
H. Sahin, ${ }^{1, *}$ J. Sivek, ${ }^{1}$ S. Li, ${ }^{1,2}$ B. Partoens, ${ }^{1}$ and F. M. Peeters ${ }^{1}$ \\ ${ }^{1}$ Department of Physics, University of Antwerp, Groenenborgerlaan 171, B-2020 Antwerpen, Belgium \\ ${ }^{2}$ Nano Structural Materials Center, Nanjing University of Science and Technology, Nanjing, China
}

(Received 26 May 2013; published 22 July 2013)

\begin{abstract}
During the synthesis of ultrathin materials with hexagonal lattice structure Stone-Wales (SW) type of defects are quite likely to be formed and the existence of such topological defects in the graphenelike structures results in dramatic changes of their electronic and mechanical properties. Here we investigate the formation and reactivity of such SW defects in silicene. We report the energy barrier for the formation of SW defects in freestanding $(\sim 2.4 \mathrm{eV})$ and $\mathrm{Ag}(111)$-supported $(\sim 2.8 \mathrm{eV})$ silicene and found it to be significantly lower than in graphene $(\sim 9.2 \mathrm{eV})$. Moreover, the buckled nature of silicene provides a large energy barrier for the healing of the SW defect and therefore defective silicene is stable even at high temperatures. Silicene with SW defects is semiconducting with a direct band gap of $0.02 \mathrm{eV}$ and this value depends on the concentration of defects. Furthermore, nitrogen substitution in SW-defected silicene shows that the defect lattice sites are the least preferable substitution locations for the $\mathrm{N}$ atoms. Our findings show the easy formation of SW defects in silicene and also provide a guideline for band gap engineering in silicene-based materials through such defects.
\end{abstract}

DOI: 10.1103/PhysRevB.88.045434

PACS number(s): 75.70.Ak, 63.22.Np, 61.72.-y, 68.35.Dv

\section{INTRODUCTION}

The electronic, thermal, and mechanical properties of graphene ${ }^{1-6}$ are sensitive to lattice imperfections and adatoms. However, the creation of in-plane defects, such as vacancies, adsorption, and doping of foreign atoms on the honeycomb structure opens the possibility for tailoring the electronic and magnetic properties of graphene-based structures. ${ }^{7-13}$ It was demonstrated that Stone-Wales (SW) defects ${ }^{14}$ can be formed during a rapid quench from high temperature or when graphene is under irradiation. ${ }^{15} \mathrm{SW}$ defects have been predicted to open a band gap in the electronic band structure that can be of potential use in the design of transistors. ${ }^{16-19}$ Moreover, the presence of such defects modifies the chemical reactivity of the graphene lattice. ${ }^{20,21}$ Very recently, Ijäs et al. have reported that the presence of SW defects in honeycomb lattice results in more reactive domains for chlorine atoms as compared to defect-free graphitic structures. ${ }^{22}$

Nowadays, active research on ultrathin materials has also been directed towards the synthesis and manipulation of monolayer silicon called silicene. In a silicene structure a hexagonal mesh of silicon atoms is buckled and studies show that its electronic properties are similar to those of graphene. ${ }^{23-25}$ After early theoretical predictions very recent experimental studies have revealed clear evidence of the existence of monolayer honeycomb structures of silicene. In recent works of Vogt et al. ${ }^{26}$ and Feng et al. ${ }^{27}$ high-quality large-scale silicene films have been synthesized on the $\mathrm{Ag}(111)$ surface. Moreover, Fleurence et al. demonstrated the successful growth of epitaxial silicene on a diboride surface. ${ }^{28}$ Recent theoretical studies have also reported that, prior to graphene, silicene has unique features such as a large spin-orbit gap at the Dirac point, ${ }^{29}$ experimentally accessible quantum spin Hall effect, ${ }^{30}$ electrically tunable band gap $^{31}$ and the emergence of a valley-polarized metal phase. ${ }^{32}$ Most recently we reported the effect of impurities on the structural, electronic, and magnetic properties and lattice dynamics of silicene. ${ }^{33,34}$

Although the SW-type defects are quite likely to be formed in such monolayer systems, the formation of SW-type defects and their effect on the electronic, magnetic, and adsorption characteristics of monolayer silicene have remained unexplored. In this paper, we use first-principles calculations within the density functional theory (DFT) formalism to investigate the formation and the electronic properties of SW defects in silicene. We found that formation of SW type defects are more likely in silicene than in graphene and the existence of the underlying $\operatorname{Ag}(111)$ surface does not significantly affect their formation. Furthermore, once the SW defect is formed in the silicene lattice the doping characteristics are influenced dramatically.

This paper is organized as follows. Section II describes the computational methodology. Section III A discusses the formation and stability of SW defects in silicene. Section III $\mathrm{B}$ presents the energetics of $\mathrm{N}$ doping in SW-defected and defect-free silicene. Findings are concluded in Sec. IV.

\section{COMPUTATIONAL METHODOLOGY}

In the present work, we performed first-principles calculations based on the plane-wave basis set with a cutoff energy of $500 \mathrm{eV}$ and the projector-augmented wave (PAW) pseudopotentials implemented in the Vienna $A b$ Initio Simulation Package (VASP). ${ }^{35,36}$ The exchange-correlation functionals are described by the generalized gradient approximation (GGA) with Perdew-Burke-Ernzerhof (PBE) approach. ${ }^{37}$ The partial occupancies for the total energy ground-state calculations were calculated with the tetrahedron methodology with Blöchl corrections. $^{38}$

Periodic boundary conditions were employed for silicene with a vacuum region of $15 \AA$ between neighboring slabs. For supported silicene on a $\operatorname{Ag}(111)$ surface the height of the supercell has been set to $29 \AA$ in order to conveniently include four layers of the Ag crystal. In order to determine the equilibrium configuration of silicene with defects, we relaxed all the atomic coordinates and the supercell geometry using the conjugate gradient $(\mathrm{CG})$ algorithm with the maximum residual forces of less than $0.01 \mathrm{eV} / \AA$. The sampling of the Brillouin zone was done for the supercell with the equivalent 
TABLE I. Calculated structural and electronic properties for silicene and SW-defected silicene: lattice parameter $(a)$, Si-Si distance $\left(d_{\mathrm{Si}-\mathrm{Si}}\right)$, angle between two of the lattice vectors of a $6 \times 6$ supercell $(\alpha)$, total magnetic moment of the system $\left(\mu_{\mathrm{Tot}}\right)$, formation energy $\left(E_{f}\right)$, cohesive energy $\left(E_{\mathrm{coh}}\right)$, and the band gap $\left(E_{\mathrm{gap}}\right)$.

\begin{tabular}{lccccccc}
\hline \hline & $\begin{array}{c}a \\
\AA\end{array}$ & $\begin{array}{c}d_{\mathrm{Si}-\mathrm{Si}} \\
\AA\end{array}$ & $\begin{array}{c}\alpha \\
\mathrm{deg}\end{array}$ & $\begin{array}{c}\mu_{\mathrm{Tot}} \\
\left(\mu_{B}\right)\end{array}$ & $\begin{array}{c}E_{f} \\
(\mathrm{eV})\end{array}$ & $\begin{array}{c}E_{\mathrm{coh}} \\
(\mathrm{eV})\end{array}$ & $\begin{array}{c}E_{\mathrm{gap}} \\
(\mathrm{meV})\end{array}$ \\
\hline Silicene & 23.21 & 2.28 & 60.0 & 0 & 0.00 & -3.98 & 1.5 \\
SW-Silicene & 23.04 & $2.19-2.28$ & 61.5 & 0 & 1.64 & -3.96 & 20 \\
\hline \hline
\end{tabular}

of $24 \times 24 \times 1$ Monkhorst-Pack ${ }^{39} k$-point mesh for a silicene unit cell composed of two $\mathrm{Si}$ atoms. In order to minimize the interactions between the neighboring SW defects, for the calculation of geometric and electronic properties, we employed a large supercell derived from a $6 \times 6$ supercell of silicene where the distance between neighboring defects is at least $14.6 \AA$. The calculations of the formation energetics of a SW defect for supported silicene were performed with a $6 \times 6$ supercell of silicene on top of $8 \times 8$ supercell of $\operatorname{Ag}(111)$ surface.

\section{RESULTS AND DISCUSSION}

\section{A. Formation and stability of Stone-Wales defects in silicene}

Since the formation of defects is inevitable in crystals, understanding their effect on the mechanical, electronic, and structural characteristics plays a key role in nanoscale device applications. The most common defects that have been observed in low-dimensional graphenelike structures are missing atoms, adatom impurities, and SW type of disorders. Here we investigate the formation and stability of SW defects in a single layer structure of silicene shown in Fig. 1(a). Compared to the graphitic materials such as graphite, graphene, and carbon nanotubes, the interatomic distance is larger in silicene. Furthermore, due to the buckled hexagonal lattice structure, easier formation of various defects in silicene can be expected.

As shown in Fig. 1(b) a SW defect can be created by the rotation of a silicon dimer by $90^{\circ}$ around the center of the Si-Si bond. From the experimental point of view, such a defect can be formed during the growth process or upon application of irradiation. After the formation of the SW defect, four neighboring hexagons of silicene are transformed into a pentagon and a heptagon pair. As can be seen from Fig. 1(b), even after the creation of the SW defect, silicene maintains its buckled two-dimensional structure with only a local out-of-plane displacement of the Si atoms. Conversely, large local bumps in the graphene lattice are formed due to the existence of SW defects. Through $90^{\circ}$ rotation of a dimer, the $\mathrm{Si}-\mathrm{Si}$ bond becomes stronger than in defect-free silicene and its length decreases from 2.28 to $2.19 \AA$. After the shortening of the bond lengths along the direction parallel to the pentagons, the lattice constant decreases from 23.21 to $23.04 \AA$. Though $\mathrm{Si}$ atoms favor buckled configuration in bare silicene, the unbuckled Si dimer in the core of the SW defect is preferable. Structural properties, energetics and energy band gap of bare and SW-defected silicene are given in Table I.
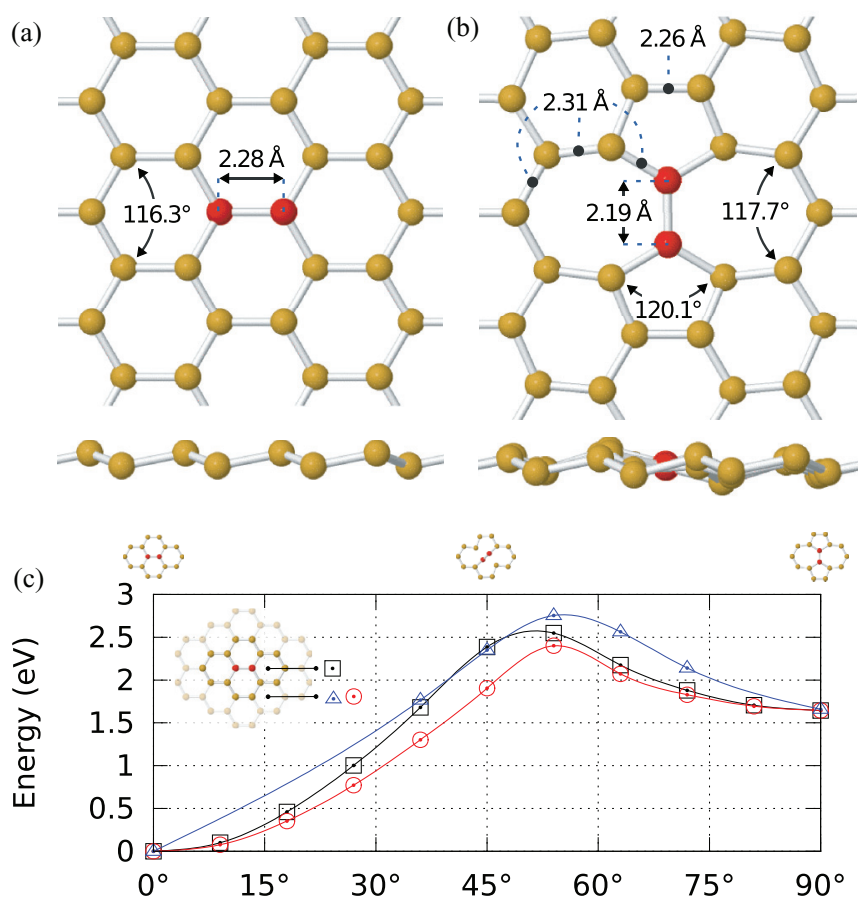

(d)
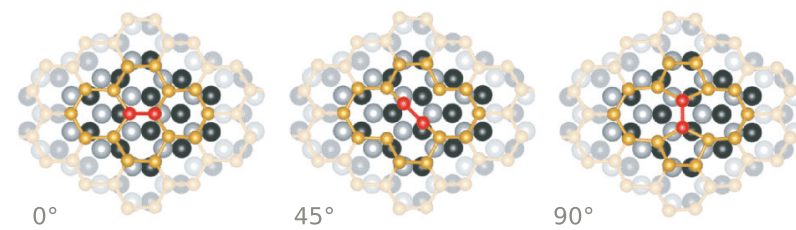

FIG. 1. (Color online) The top and side view of (a) perfect and (b) SW-defected silicene. (c) Energetics of formation of a SW defect in silicene via the bond rotation (highlighted). The black (open squares) curve is obtained by relaxing the first-nearest hexagons to the silicene dimer in freestanding silicene layer. Red (open circles) and blue (open triangles) curves are obtained by relaxing up to the second-nearest hexagons to the silicon dimer in the freestanding and supported silicene [on a $\operatorname{Ag}(111)$ surface], respectively. (d) The actual bond rotation steps $\left(0^{\circ}, 45^{\circ}\right.$, and $\left.90^{\circ}\right)$ in silicene supported on the $\mathrm{Ag}(111)$ surface. The uppermost and lower-lying Ag atoms are shown by gray (light) and black (dark).

The transition states and energetics of the formation steps of the SW defect in freestanding silicene as well as for silicene supported on the $\operatorname{Ag}(111)$ surface are shown in Fig. 1(c). The intermediate steps are obtained by confining $\mathrm{Si}$ atoms at positions interpolated between those of perfect silicene and silicene with a SW defect. The atoms that form two heptagons and two pentagons of the SW defect and the nearest hexagons are allowed to relax. The $\mathrm{Si}$ dimer that is gradually rotated is allowed to relax only in the direction perpendicular to the Si layer. It is seen that the formation of such SW defect in a perfect silicene lattice may occur by overcoming the energy barrier of $\sim 2.4 \mathrm{eV}$ for freestanding silicene and $2.8 \mathrm{eV}$ for the supported silicene layer. The maximum of the energy barrier corresponds to the $\sim 54^{\circ}$ rotation of the bond. In comparison, this barrier in graphitic materials is considerably larger and amounts to $9.2 \mathrm{eV}^{20}$ However, the energy barrier of $0.76 \mathrm{eV}$ (1.10 eV for supported silicene) between the intermediate configuration and silicene with a SW defect guarantees the 
stability of such defects even at high temperatures. This shows that the buckled lattice structure and the softer bonding nature of silicene allows an easier formation of SW-defected structure. The formation energy, $E_{f}$, of a SW defect in silicene is given by

$$
E_{f}=E_{\text {Silicene }}^{\mathrm{SW}}-E_{\text {Silicene }},
$$

where $E_{\text {Silicene }}^{\mathrm{SW}}$ is the total energy of silicene with SW defect and $E_{\text {Silicene }}$ is the total energy of perfect silicene. According to this, the formation energy of a SW defect in a $6 \times 6 \times 1$ supercell is calculated to be $1.64 \mathrm{eV}$. Additionally, the stability of the structure can also be predicted via its cohesive energy which is defined as the energy required for separating the crystal into isolated free atoms.

The cohesive energy of a crystal structure is given by the formula

$$
E_{\mathrm{coh}}=\left(E_{\mathrm{Tot}} / n\right)-E_{\mathrm{Si}},
$$

where $E_{\text {Tot }}$ is the total energy of the system, $n$ is the number of atoms in the supercell and $E_{\mathrm{Si}}$ is the total energy of an isolated silicon atom. The cohesive energies of silicene and SW-defected silicene are calculated to be -3.98 and $-3.96 \mathrm{eV}$, respectively. The negative cohesive energies of both structures indicate their stability.

Next we extend our discussion on the stability and formation of SW-type defects in single layer silicene by analyzing the vibrational spectrum. Although total energy calculations show the stability of the SW defects, our phonon calculations reveal that there are two imaginary eigenfrequencies in the SWdefected silicene spectrum (see Fig. 2). For a $4 \times 4$ supercell of silicene these modes are located at -14 and $-20 \mathrm{~cm}^{-1}$. Analysis of the lattice dynamics of structure for these two modes shows that the creation of a SW defect results in large ripples around the defected region. As seen from Fig. 2, the modes I and II imply the formation of sinelike and cosinelike lattice distortions with respect to the midpoint of the $\mathrm{Si}-\mathrm{Si}$ dimer. Therefore the buckled planar structure of SW-defected
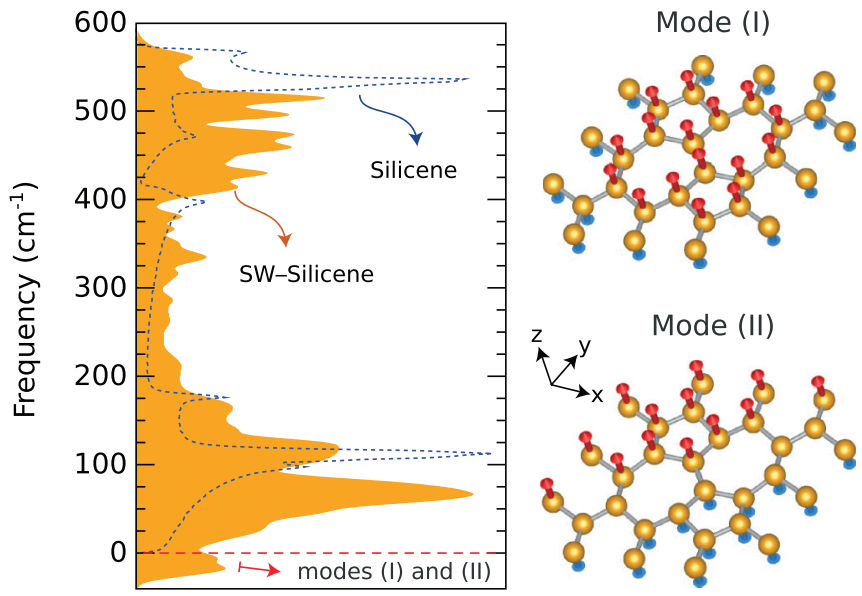

FIG. 2. (Color online) Phonon dispersion of pure (dashed line) and SW-defected silicene (filled curve). Atomic motions for two phonon modes, which have imaginary eigenfrequencies, are shown on the right. Only the motions of atoms in defect core and neighboring atoms are shown for clarity. Blue and red arrows represent displacements of $\mathrm{Si}$ atoms along $-z$ and $+z$ directions, respectively. (a) Silicene

(b) SW silicene
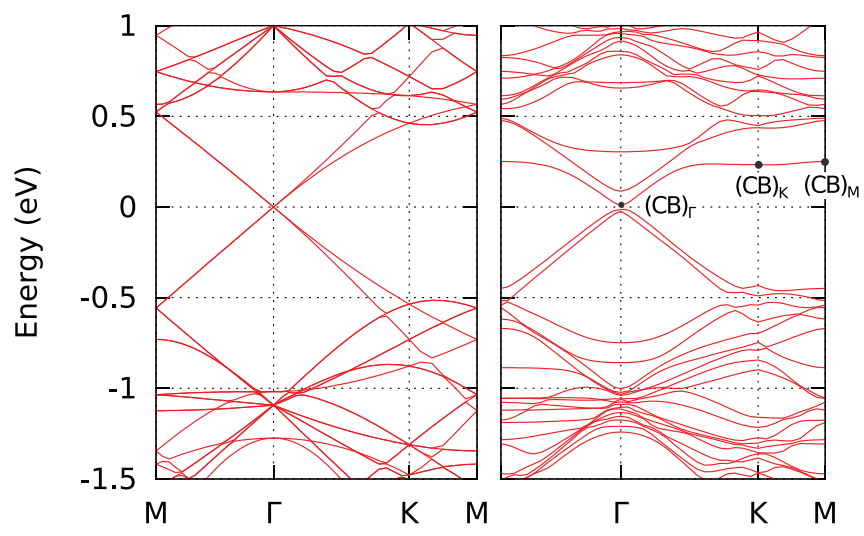

(c)

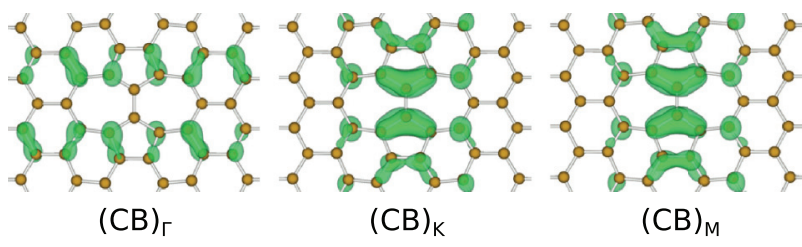

FIG. 3. (Color online) The electronic band structure for a $6 \times 6$ supercell of (a) perfect and (b) SW-defected silicene. (c) Band decomposed charge densities of the conduction band around the Fermi level at $\Gamma, K$, and $M$ symmetry points (the isosurface is set to $\left.0.57 \times 10^{-3} \mathrm{e} / \AA^{3}\right)$

silicene is a local minimum on the potential energy surface. However, it is worth noting that the stabilization of planar silicene structure by a supporting surface has already been reported by several groups. ${ }^{26-28}$ The existence of sinelike and cosinelike rippling in silicene is in good agreement with the results for SW-defected graphene. ${ }^{18}$ Although our phonon calculations revealed the instability of densely SW-defected silicene, the results also imply the stability provided by the long-wavelength ripples. Therefore it is reasonable to assume that the reactivity of defect cores for planar and long-wavelength-rippled silicene do not differ significantly. Additionally, we perform molecular dynamics calculations in order to further examine the thermal stability of SW-defected silicene. At the temperature of $500 \mathrm{~K}$, we choose time steps of 1 and also 2 fs. The SW defect and the surrounding bonds in silicene remain stable and there is no indication of defect healing throughout the molecular dynamics simulations with duration of $2 \mathrm{ps}$ at $500 \mathrm{~K}$.

In Figs. 3(a) and 3(b) the electronic band dispersion of perfect and SW-defected silicene are presented. It is worth noting that for a $6 \times 6$ supercell, the $K$ and $K^{\prime}$ symmetry points of the unit cell of silicene are folded onto the $\Gamma$ point. Therefore, both valence band maximums are transferred to the $\Gamma$ point of the supercell. Clearly, as seen in Fig. 1(b), after the formation of a SW defect the lattice symmetry of silicene is broken. Since the formation of the SW defect breaks the sixfold symmetry of the silicene lattice, which results in linearly crossing bands and the existence of highly mobile fermions in the vicinity of the Dirac point, a band gap opening occurs at the crossing point. ${ }^{40}$ The band gap opening for SW-defected silicene in a $6 \times 6$ supercell is calculated to be $0.02 \mathrm{eV}$. It corresponds to the system with a defect concentration of $1.9 \times 10^{13} \mathrm{~cm}^{-2}$. The 
actual value of the band gap depends on the concentration of the SW defects. In the case of a $5 \times 5$ supercell with a single SW defect $\left(2.7 \times 10^{13} \mathrm{~cm}^{-2}\right)$ the energy band gap increases to $0.1 \mathrm{eV}$. Here it is also worth noting that the choice of a hexagonal supercell results in band edges that appear at the $K$ symmetry point ( $\Gamma$ for $3 n \times 3 n$ supercells) and a temporarily ordered defect configuration may result in a different band dispersion. Since there are no dangling bonds introduced into the silicene lattice with the creation of a SW defect, all the atomic orbitals of the $\mathrm{Si}$ atoms at the vicinity of the defect are paired and hence there is no defect-originated magnetism.

\section{B. N-doped silicene: Effect of Stone-Wales defects}

Doping materials with foreign atoms is an efficient way to manipulate their electronic, magnetic, and chemical properties. For carbon-based materials such as graphite, graphene, and nanotubes the doping especially with Group III or V atoms is desirable since they induce $n$ or $p$ type doping and the density of the charge carriers can be tuned by the concentration of the substituents. It has been demonstrated that synthesis of $\mathrm{N}$-doped graphene samples can be achieved by annealing in the presence of pyridine or $\mathrm{NH}_{3}$ molecules and by chemical vapor deposition (CVD) and the observed features of $\mathrm{N}$-doped graphene are quite desirable for device applications. ${ }^{41-45}$ The formation energy of $\mathrm{N}$ substitution in graphene is $0.32 \mathrm{eV}$ per $\mathrm{N}$ atom. ${ }^{46}$ Since $\mathrm{N}$ atom in graphene forms three $\sigma$ bonds and $p_{z}$ orbital is filled by two electrons, $\mathrm{N}$-doped graphene shows nonmagnetic behavior. ${ }^{41}$ The $\mathrm{N}$ doping induces $n$-type doping of graphene and shifts the Fermi level up by $\sim 0.6 \mathrm{eV}$ for the case of $2 \% \mathrm{~N}$ concentration. ${ }^{42}$

The substitutional doping of silicene with a $\mathrm{N}$ atom is of a different nature as compared to the doping of graphene. The N-Si bond length in substituted silicene (1.83 $\AA$ ) is significantly shorter than the $\mathrm{Si}-\mathrm{Si}$ bond length in pristine silicene $(2.28 \AA)$ and the shorter bonds induce a local in plane shrink deformation in the structure as presented in Fig. 4(b).

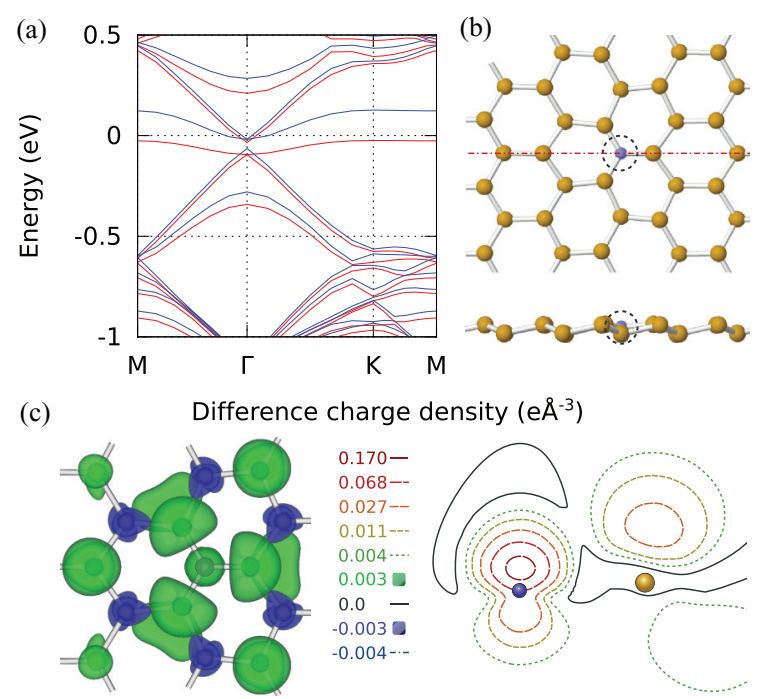

FIG. 4. (Color online) (a) The electronic band structure of Ndoped silicene, (b) top and side view of the structure. (c) 3D and contour plot of difference charge density $\left(\rho_{\uparrow}-\rho_{\downarrow}\right)$. The slicing plane is marked by dot-dashed line in (b).
The nitrogen bonds with the neighboring $\mathrm{Si}$ atoms are in the plane, which reduces the amount of local buckling. However, perfect silicene's lattice structure is restored just several bonds away from the substituent atom. The formation energy of $\mathrm{N}$-doping in silicene is defined as

$$
E_{f}=E_{\mathrm{Tot}}-\left(E_{\text {Silicene }}-\mu_{\mathrm{Si}}\right)-\mu_{N},
$$

where $E_{\mathrm{Tot}}$ is the total energy of the N-doped silicene and $E_{\text {Silicene }}$ is the total energy of perfect silicene, $\mu_{\mathrm{Si}}$ is the chemical potential of a single silicon atom (calculated from the total energy of monolayer silicene), and $\mu_{N}$ is the chemical potential of a nitrogen atom, defined as one-half of the total energy of the $N_{2}$ molecule in the gas phase. This choice of chemical potentials is made to account for the stability of $\mathrm{N}$-doped silicene against $N_{2}$ molecular desorption. The binding energy of $\mathrm{N}$-doped silicene is here defined as

$$
E_{\text {bind }}=E_{\text {Tot }}-\left(E_{\text {Silicene }}\left(n_{\mathrm{Si}}-1\right) / n_{\mathrm{Si}}\right)-E_{N},
$$

where $n_{\mathrm{Si}}$ is the number of $\mathrm{Si}$ atoms in a supercell of pristine silicene and $E_{N}$ is the total energy of an isolated $\mathrm{N}$ atom. The binding energy of a $\mathrm{N}$ atom in $\mathrm{N}$-doped silicene is $-4.60 \mathrm{eV}$. On the other hand, the calculated formation energy is $0.59 \mathrm{eV}$ as a consequence of the large bond-dissociation energy of the $\mathrm{N}_{2}$ molecule.

From the electronic band structure of $\mathrm{N}$-doped silicene, displayed in Fig. 4(a), it is seen that the substitutional $\mathrm{N}$ atom turns semimetallic silicene into a ferromagnetic metal with total magnetic moment of $1.0 \mu_{B}$. The magnetic character of $\mathrm{N}$ substituted silicene can be seen from the $3 \mathrm{D}$ and the contour plot of the difference charge density $\left(\rho_{\uparrow}-\rho_{\downarrow}\right)$ in Fig. 4(c). It appears that the $\mathrm{N}$-originated state is delocalized over the nearest $\mathrm{Si}$ atoms and therefore $\mathrm{N}$ substitution results in longrange spin polarization of the surrounding $s p^{3}$-like orbitals even in a large $6 \times 6$ supercell. The magnetic behavior of $\mathrm{N}$ doped silicene and delocalization of the N-originated states are consistent with previously reported results obtained with the use of LDA functionals. ${ }^{33,34}$

Next we investigate the effect of the presence of a SW defect on substitutional nitrogen doping in silicene. As a result of local deformations induced by the creation of a SW defect [Fig. 1(b)] every single Si atom in the surroundings of a SW defect provides a unique place for substitution. Since the surrounding atoms still maintain their buckled hexagonal nature we will focus only on the Si atoms that are part of the heptagons or pentagons in the SW defect. As a consequence of the lattice symmetry with respect to the Si-Si dimer at the defect core a similar behavior of adsorbates on site I and II can be expected. However, in order to not exclude the possible effects of lattice distortion on $\mathrm{N}$ substitution, calculations were performed for all 16 sites of the SW defect. Possible positions for substitutional doping with one substituent atom are displayed in Fig. 5. Additionally, we present structural properties, total magnetic moment, formation energies, and binding energy values in Table II.

The substitution positions can be divided into the four groups. The first group contains the lattice sites that belong to edges of heptagons $\mathrm{A}, \mathrm{B}, \mathrm{H}$, and $\mathrm{I}$. We found that $\mathrm{Si}-\mathrm{N}$ bonds at the edges of heptagons are not stable and hence the substitution of a $\mathrm{N}$ atom on $\mathrm{A}, \mathrm{B}, \mathrm{H}$, and I positions cannot be realized. 


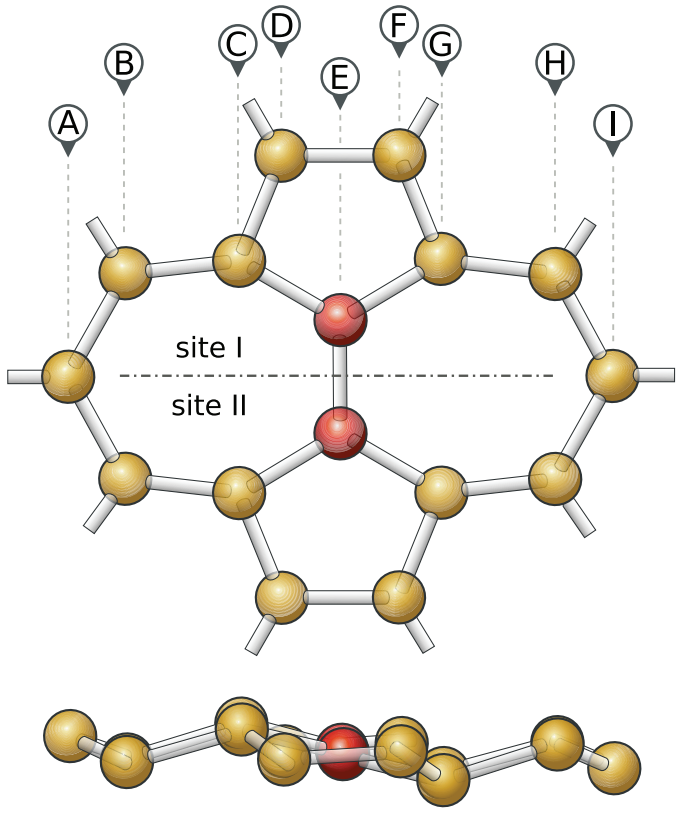

FIG. 5. (Color online) The substitutional sites of the SW defect in silicene with associated letter, and the side view of the same structure.

The second group is composed of C and G substitution sites that locate at the outermost intersection of heptagons and the pentagons of the defect core. Though $\mathrm{C}$ and $\mathrm{G}$ sites are geometrically and energetically similar, because of the lattice distortion induced by $\mathrm{N}$ doping and buckled nature of the silicene lattice, these adsorption sites are not identical. Similar to doping in perfect silicene the substitution of a $\mathrm{N}$ atom on $\mathrm{C}$ and $\mathrm{G}$ site result in spin polarization of $1 \mu_{B}$. The binding energies on these lattice sites are smaller compared to intrinsic silicene.

The third group of possible substitution points are I and II sides of $\mathrm{E}$, which are located in the middle of the defect core. Clearly, both sides of $\mathrm{E}$ are equal for $\mathrm{N}$-doping. When a $\mathrm{N}$ atom is substituted over $\mathrm{E}$ site, the resulting structure has a total magnetic moment of $1 \mu_{B}$ and doping on these sites is by $0.4 \mathrm{eV}$ more favorable than doping on $\mathrm{C}$ and $\mathrm{G}$ sites.

The last group includes D and F substitution sites, which belong to the pentagons of the SW defect. The Si-N bond lengths in these lattice sites are smaller or equal to the bond

TABLE II. Calculated values for N-doped SW-defected $6 \times 6$ silicene; bond length of $\mathrm{N}-\mathrm{Si}\left(d_{\mathrm{N}-\mathrm{Si}}\right)$, angle between two of the lattice vectors of supercell of $\mathrm{N}$-doped structure $(\alpha)$, total magnetic moment of the system $\left(\mu_{\mathrm{Tot}}\right)$, formation energy $\left(E_{f}\right)$, and binding energy ( $\left.E_{\text {bind }}\right)$.

\begin{tabular}{lccccc}
\hline \hline Defect site & $\begin{array}{c}d_{\mathrm{N}-\mathrm{Si}} \\
(\AA)\end{array}$ & $\begin{array}{c}\alpha \\
(\mathrm{deg})\end{array}$ & $\begin{array}{c}\mu_{\mathrm{Tot}} \\
\left(\mu_{B}\right)\end{array}$ & $\begin{array}{c}E_{f} \\
(\mathrm{eV})\end{array}$ & $\begin{array}{c}E_{\text {bind }} \\
(\mathrm{eV})\end{array}$ \\
\hline N @ pure silicene & 1.831 .831 .83 & 60.0 & 1.0 & 0.59 & -4.6 \\
N @ A,B,H,I & - & - & - & - & - \\
N @ C & 1.861 .821 .80 & 61.7 & 1.0 & 1.8 & -3.4 \\
N @ D & 1.781 .821 .80 & 61.5 & 0.7 & 1.1 & -4.0 \\
N @ E & 1.831 .831 .76 & 61.3 & 1.0 & 1.5 & -3.7 \\
N @ F & 1.801 .781 .82 & 61.5 & 0.7 & 1.1 & -4.0 \\
N @ G & 1.791 .821 .83 & 61.7 & 1.0 & 1.8 & -3.3 \\
\hline \hline
\end{tabular}

lengths in $\mathrm{N}$-doped silicene. For those structures we also observe large rippling of the order of $2 \AA$. We found that substitutional doping on $\mathrm{D}$ and $\mathrm{F}$ positions does not give rise to a shrinkage of the lattice parameters. $\mathrm{D}$ and $\mathrm{F}$ points are energetically most favorable sites on a SW defect with a binding energy of $-4.0 \mathrm{eV}$. It is seen that nitrogen substitution results in a net magnetic moment of $0.7 \mu_{B}$. Similar to $\mathrm{N}$ doping in perfect silicene, SW-defected silicene shows metallic behavior for all possible substitution sites that belong to the core of SW defect.

\section{CONCLUSION}

In this study we performed ab initio calculations in order to investigate the formation of SW defects in silicene and to show the effect of these defects on the reactivity of the silicene with respect to nitrogen doping. We found the formation of a SW defect to be much easier in the buckled lattice structure of silicene as compared to graphene. The softer bonding nature of silicene allows easier formation of a SW defect with a smaller energy barrier for both freestanding as well as supported silicene. The presence of a SW defect in silicene breaks the symmetry and results in a band gap opening in electronic band structure with size depending on the defect concentration. Additionally, our vibrational analysis reveals that the formation of SW defects in freestanding silicene inevitably leads to the formation of large ripples. Moreover, the presence of an underlying $\operatorname{Ag}(111)$ supporting surface increases the barrier for the formation of SW defects from 2.4 to $2.8 \mathrm{eV}$. We also found that the buckled nature of silicene provides a large energy barrier for healing of SW defect and therefore defective silicene is quite stable even at high temperatures.

Furthermore, the presence of SW defects significantly modifies the doping characteristics of silicene. While doping defect-free silicene by $\mathrm{N}$ atoms is favorable with a little lattice shrinking, the presence of a SW defect limits the number of possible doping sites. We found that all the nitrogen substitution sites on the defect core are less preferable than on defect-free silicene. Among the possible sites of defect the edges of heptagons are least favorable sites while doping of the $\mathrm{N}$ atoms at the edges of pentagons is most preferable. Our findings on the reactivity of SW-defected silicene domains agree well with the very recent study that reports the chlorine adsorption on SW-defected graphene and carbon nanotube. ${ }^{22}$ We believe that our results provide a basis for the understanding of the characteristic properties of defected silicene, which are essential for its utilization in future electronics.

\section{ACKNOWLEDGMENTS}

This work was supported by the Flemish Science Foundation (FWO-Vl) and the Methusalem foundation of the Flemish government. Computational resources were provided by TUBITAK ULAKBIM, High Performance and Grid Computing Center (TR-Grid e-Infrastructure), and HPC infrastructure of the University of Antwerp (CalcUA) a division of the Flemish Supercomputer Center (VSC), which is funded by the Hercules foundation. H.S. is supported by a FWO Pegasus Marie Curie Fellowship. 
*hasan.sahin@ua.ac.be

${ }^{1}$ K. S. Novoselov, A. K. Geim, S. V. Morozov, D. Jiang, Y. Zhang, S. V. Dubonos, I. V. Grigorieva, and A. A. Firsov, Science 306, 666 (2004).

${ }^{2}$ S. V. Morozov, K. S. Novoselov, M. I. Katsnelson, F. Schedin, D. C. Elias, J. A. Jaszczak, and A. K. Geim, Phys. Rev. Lett. 100, 016602 (2008).

${ }^{3}$ J. H. Chen, C. Jiang, S. Xiao, M. Ishigami, and M. S. Fuhrer, Nature Nanotechnol. 3, 206 (2008).

${ }^{4}$ A. A. Balandin, S. Ghosh, W. Bao, I. Calizo, D. Teweldbrhan, F. Miao, and C. N. Lau, Nano Lett. 8, 902 (2008).

${ }^{5}$ C. Lee, X. Wei, J. W. Kysar, and J. Hone, Science 321, 385 (2008). ${ }^{6}$ S. Cahangirov, C. Ataca, M. Topsakal, H. Sahin, and S. Ciraci, Phys. Rev. Lett. 108, 126103 (2012).

${ }^{7}$ F. Banhart, J. Kotakoski, and A. V. Krasheninnikov, ACS Nano 5, 26 (2011).

${ }^{8}$ R. Balog, B. Jorgensen, L. Nilsson, M. Andersen, E. Rienks, M. Bianchi, M. Fanetti, E. Laegsgaard, A. Baraldi, S. Lizzit, Z. Sljivancanin, F. Besenbacher, B. Hammer, T. G. Pedersen, P. Hofmann, and L. Hornekaer, Nature Mater. 9, 315 (2010).

${ }^{9}$ J. Lahiri, Y. Lin, P. Bozkurt, I. I. Oleynik, and M. Batzill, Nature Nanotech. 5, 326 (2010).

${ }^{10}$ L. Tapasztó, G. Dobrik, P. Nemes-Incze, G. Vertesy, Ph. Lambin, and L. P. Biró, Phys. Rev. B 78, 233407 (2008).

${ }^{11}$ A. V. Krasheninnikov and F. Banhart, Nature Mater. 6, 723 (2007).

${ }^{12}$ M. H. Gass, U. Bangert, A. Bleloch, P. Wang, R. R. Nair, and A. K. Germ, Nature Nanotechnol. 3, 676 (2008).

${ }^{13}$ M. Topsakal, H. Sahin, and S. Ciraci, Phys. Rev. B 85, 155445 (2012).

${ }^{14}$ A. J. Stone and D. J. Wales, Chem. Phys. Lett. 128, 501 (1986).

${ }^{15}$ J. C. Meyer, C. Kisielowski, R. Erni, M. D. Rossell, M. F. Crommie, and A. Zettl, Nano Lett. 8, 3582 (2008).

${ }^{16}$ Georgii G. Samsonidze, Guram G. Samsonidze, and B. I. Yakobson, Phys. Rev. Lett. 88, 065501 (2002).

${ }^{17}$ C. P. Ewels et al., Chem. Phys. Lett. 351, 178 (2002).

${ }^{18}$ J. Ma, D. Alfe, A. Michaelides, and E. Wang, Phys. Rev. B 80, 033407 (2009).

${ }^{19}$ M. T. Lusk and L. D. Carr, Phys. Rev. Lett. 100, 175503 (2008).

${ }^{20}$ Y. Li, J. C. Ren, R. Q. Zhang, Z. J. Lin, and M. A. Van Hove, J. Mater. Chem. 22, 21167 (2012).

${ }^{21}$ L. Chen, H. Hu, Y. Ouyang, H. Z. Pan, Y. Y. Sun, and F. Liu, Carbon 49, 3356 (2011).

${ }^{22}$ M. Ijäs, P. Havu, and A. Harju, Phys. Rev. B 87, 205430 (2013).

${ }^{23}$ K. Takeda and K. Shiraishi, Phys. Rev. B 50, 14916 (1994).
${ }^{24}$ S. Cahangirov, M. Topsakal, E. Akturk, H. Sahin, and S. Ciraci, Phys. Rev. Lett. 102, 236804 (2009).

${ }^{25}$ H. Sahin, S. Cahangirov, M. Topsakal, E. Bekaroglu, E. Akturk, R. T. Senger, and S. Ciraci, Phys. Rev. B 80, 155453 (2009).

${ }^{26}$ P. Vogt, P. De Padova, C. Quaresima, J. Avila, E. Frantzeskakis, M. C. Asensio, A. Resta, B. Ealet, and G. LeLay, Phys. Rev. Lett. 108, 155501 (2012)

${ }^{27}$ B. J. Feng, Z. J. Ding, S. Meng, Y. G. Yao, X. Y. He, P. Cheng, L. Chen, and K. H. Wu, Nano Lett. 12, 3507 (2012).

${ }^{28}$ A. Fleurence, R. Friedlein, T. Ozaki, H. Kawai, Y. Wang, and Y. Yamada-Takamura, Phys. Rev. Lett. 108, 245501 (2012).

${ }^{29}$ Cheng-Cheng Liu, H. Jiang, and Y. Yao, Phys. Rev. B 84, 195430 (2011).

${ }^{30}$ Cheng-Cheng Liu, W. Feng, and Y. Yao, Phys. Rev. Lett. 107, 076802 (2011)

${ }^{31}$ N. D. Drummond and V. Zolyomi, and V. I. Fal'ko, Phys. Rev. B 85, 075423 (2012).

${ }^{32}$ M. Ezawa, Phys. Rev. Lett. 109, 055502 (2012).

${ }^{33}$ H. Sahin and F. M. Peeters, Phys. Rev. B 87, 085423 (2013).

${ }^{34}$ J. Sivek, H. Sahin, B. Partoens, and F. M. Peeters, Phys. Rev. B 87, 085444 (2013).

${ }^{35}$ G. Kresse and J. Furthmuller, Comput. Mater. Sci. 6, 15 (1996).

${ }^{36}$ G. Kresse and D. Joubert, Phys. Rev. B 59, 1758 (1999).

${ }^{37}$ J. P. Perdew, K. Burke, and M. Ernzerhof, Phys. Rev. Lett. 77, 3865 (1996).

${ }^{38}$ P. E. Blöchl, O. Jepsen, and O. K. Andersen, Phys. Rev. B 49, 16223 (1994).

${ }^{39}$ H. J. Monkhorst and J. D. Pack, Phys. Rev. B 13, 5188 (1976).

${ }^{40}$ H. Sahin and S. Ciraci, Phys. Rev. B 84, 035452 (2011).

${ }^{41}$ S. Kattel, P. Atanassov, and B. Liefer, J. Phys. Chem. C 116, 8161 (2012).

${ }^{42}$ L. S. Panchakarla, K. S. Subrahmanyam, S. K. Saha, A. Govindaraj, H. R. Krishnamurthy, U. V. Waghmare, and C. N. R. Rao, Adv. Mater. 21, 4726 (2009).

${ }^{43}$ L. Zhao, R. He, K. T. Rim, T. Schiros, K. S. Kim, H. Zhou, C. Gutiérrez, S. P. Chpckalingam, C. J. Arguello, L. Pálová, D. Nordlund, M. S. Hybertsen, D. R. Reichman, T. F. Heinz, P. Kim, A. Pinczuk, G. W. Flynn, and A. N. Pasupathy, Science 333, 999 (2011).

${ }^{44}$ X. R. Wang, X. L. Li, L. Zhang, Y. K. Yoon, P. K. Weber, H. L. Wang, J. Guo, and H. J. Dai, Science 324, 768 (2009).

${ }^{45}$ D. C. Wei, Y. Q. Liu, Y. Wang, H. L. Zhang, L. P. Huang, and G. Yu, Nano Lett. 9, 1752 (2009).

${ }^{46}$ Y. Fujimoto and S. Saito, Phys. Rev. B 84, 245446 (2011). 